\title{
El poder iterativo: Herbert Gladstone y el proyecto imperial británico en Sudáfrica a principios del siglo $\mathrm{xx}$
}

\author{
Iterative power: \\ Herbert Gladstone and \\ the British imperial project in \\ early twentieth century South Africa
}

ALISTAIR FRASER*

Resumen: Con base en documentos de archivo relacionados con Herbert Gladstone, primer gobernador general de la Unión Sudafricana de 1910 a 1914, se explora la adopción del poder iterativo, centrado en la inevitable falibilidad de las personas encargadas de la toma de decisiones, que responden a la disposición espacial de diversos materiales y flujos, y tratan de alterarlos. Centrándose en la interacción de Gladstone en un incidente crítico en 1914, se revela cómo imaginó alterar la geografía única del país; sin embargo, también se evidencia la manera en que el poder iterativo que él manifestó, finalmente, fue incapaz de gestionar la dinámica de la acumulación capitalista, de la cuestión racial y del proyecto imperial en Sudáfrica.

Palabras clave: Sudáfrica; Unión Sudafricana; Herbert Gladstone; historiografía; poder iterativo.

Abstract: This article uses archival documents concerning Herbert Gladstone, first Governor-General of the Union of South Africa from

Recepción: 25 de agosto de 2016. / Aceptación: 20 de julio de 2017.

* Maynooth University, alistair.fraser@nuim.ie 
1910 to 1914, to explore the enactment of iterative power. Iterative power focuses on the inevitable fallibility of individual decisionmakers who respond to, and seek to alter, the spatial arrangement of diverse materials and flows. Focusing on Gladstone's interaction with a critical incident in 1914, the article reveals how he imagined altering the country's unique geography but yet how the iterative power he expressed was ultimately incapable of negotiating the dynamics of capitalist accumulation, race, and the imperial project in South Africa.

Key words: South Africa; Union of South Africa; Herbert Gladstone; historiography; iterative power.

\section{Introducción}

En una reciente discusión sobre la interacción entre política, economía y mercados, Judith Butler advierte sobre la importancia de una "estructura iterativa" constituida por procesos y acciones múltiples y continuas que representan y recrean la vida social (Butler, 2010, pp. 147-161). Su enfoque es, en parte, sobre la manera en la que la "autonomía ostensible de la economía” (Butler, 2010, pp. 149) se produce a través de un ir y venir iterativo y performativo que distingue lo que es $y$ lo que no es económico, pero hay algo que se puede decir para enfatizar la centralidad más general de las estructuras y los procesos que pueden ser iterados [repetidos] en la construcción de la sociedad. El "poder iterativo" se refiere a esos momentos en los que los responsables de la toma de decisiones en diversos entornos se involucran en un acto performativo de ida y vuelta [recíproco/de doble vía]; cuando responden a las contradicciones, las dinámicas y las tensiones sociales; cuando experimentan reorganizando relaciones, materiales e instituciones para (intentar) producir efectos específicos (a sabiendas de que cierto tipo de fracaso resulta inevitable). Esta forma de poder no determinista reconoce la falibilidad de las decisiones y de los responsables de tomarlas, que el mundo a nuestro alrededor nos invita a realizar, pero que también restringe los movimientos y los ajustes que se podrían ingeniar cuando se comprometen con los ensamblajes de las relaciones 
sociales o tratan de alterarlos. Asimismo, se sostiene que tomar en consideración el poder iterativo exige una investigación empírica que analice el modo en que los individuos dentro de las estructuras iterables de toma de decisiones ejercen el poder, no sólo haciendo lo que se espera de ellos, sino también a través de procesos de conversión mediante los cuales el mundo social que los rodea es confrontado, interpretado, teorizado y vuelto a hacer.

Sin embargo, cuando los individuos ejercen el poder tan iterativamente - cuando recurren a distintos medios para comprender y luego tratar de intervenir con éxito en el mundo- resulta crucial reconocer cómo ocurren sus acciones en un plano geográfico, es decir, ejercer el poder iterativo siempre implica participar en un proceso de reordenamiento espacial. Para Butler, entonces, la toma de decisiones políticas se produce a través de un proceso iterativo, pero apegándose a las ideas de geógrafos como Doreen Massey (2005), que señala que dicho proceso implica teorizar sobre cómo se podrían ajustar la distribución y la posición relativa de diversos materiales. Para lograrlo, es necesario recurrir a las ideas sobre la forma en la que podrían modificarse los patrones de numerosos flujos para que surjan resultados que ya hubieran sido anticipados. En resumen, si los geógrafos tienen razón al postular que el espacio es producto de las relaciones sociales (Massey, 2005; Massey y Allen, 1995), y si las más cruciales implican prácticas de poder (a través de la seducción, la manipulación o la dominación) (Allen, 2003), se concluye que el poder siempre se ejerce de manera iterativa, por lo que necesariamente recurre al espacio, pero también lo recrea.

Por supuesto que siempre varía la manera en la que una persona encargada de la toma de decisiones se involucra en esta especie de proceso iterativo. Sin embargo, no cabe duda de que en el contexto de las sociedades en las que se produce la acumulación capitalista, tanto las contradicciones que dichos procesos de acumulación deben negociar, como las que inevitablemente crean, influyen en la toma de decisiones políticas. En contraste con una estricta interpretación marxista del capitalismo, lo que exige la provocación de Butler es reconocer que el poder del Estado, la acción en la esfera democrática o el ejercicio 
individual del poder iterativo sólo responden a la acumulación, y no necesariamente de modos que siempre encajan en la lógica capitalista. Además, cuando se piensa geográficamente en los procesos que aquí se discuten, se amplifica la necesidad de este sentido más abierto de relación entre lo político y lo económico. El punto central es que la acumulación capitalista ocurre en el ámbito geográfico, que las particularidades espaciales importan, que las soluciones y los flujos poco comunes se encuentran entre sí y son alterados y atraídos a dondequiera que se produzca la acumulación capitalista (sobre la espacialidad del capitalismo, véase especialmente Harvey, 1982). Lo que se logra es que, cuando quienes toman las decisiones calculan iterativamente la manera en la que se pueden ajustar las soluciones y los flujos, deben interpretar la dinámica y las posibilidades de la acumulación y de la geografía desigual con la que interactúan. Debido a la naturaleza abierta del espacio, y porque el futuro no está predeterminado por cómo los procesos se desarrollan hoy en día, el ejercicio del poder implica tomar decisiones que quizá más tarde resulten erróneas. Si queremos entender cómo se ejerce el poder iterativo, es esencial prestar atención al espacio - a la geografía- y a las configuraciones espaciales de los materiales y de las relaciones sociales, sobre todo cuando la toma de decisiones se ve obligada a lidiar con los procesos de acumulación capitalista (y con las contradicciones que de ella surgen).

\section{Sudáfrica en la mira}

Pocos lugares en el mundo ilustran como Sudáfrica la importancia de la geografía en la relación entre política y acumulación capitalista (Cox, 2002). La acumulación en Sudáfrica se produjo a través de la creación y el mantenimiento de una configuración única de materiales y flujos, especialmente el sistema laboral migratorio, que hizo posible la rentabilidad en las numerosas minas de oro del país. A partir de la década de 1880, bajo la fuerza de la compulsión económica, los africanos se sintieron atraídos por el Rand sudafricano y estuvieron dispuestos a trabajar durante largos periodos que, a menudo, abarcaban 
lapsos de casi un año (Callinicos, 1985). Mientras se ocuparan en las minas, tenían que vivir en los complejos habitacionales creados para los trabajadores, y durante ese tiempo sus familias seguían residiendo en las áreas rurales -que hacia principios del siglo xx, y de ahí en adelante, a través de leyes como el Acta sobre las Tierras Nativas de 1913, fueron conocidas como "reservas nativas"- (Beinart y Delius, 2014, pp. 667-688). Este sistema laboral, en conjunción con leyes racialmente discriminatorias y prácticas que reservaban el trabajo minero relativamente calificado sólo para los blancos, fue el responsable de que en Sudáfrica la minería intensiva en mano de obra fuera altamente rentable y constituyera, por lo tanto, la base económica para otras transformaciones en un país que, eventualmente, se convertiría en la potencia industrial del sur de África. Las particularidades del lugar - el encuentro de la geología, la urbanización, la raza, la ambición imperial y la universalización de las abstracciones coloniales sobre las posibilidades del "progreso" y el futuro- se combinaron con un proceso de acumulación más general. La historia de Sudáfrica está íntimamente ligada a la constante negociación iterativa de la peculiar configuración de sus relaciones socioespaciales.

La forma como los responsables de la toma de decisiones respondieron a esta geografía -esto es, cómo interpretaban la geografía de Sudáfrica que constituía la base de los cálculos sobre los procedimientos para ejercer el poder- ha sido poco investigada en la historiografía sudafricana. Esta deficiencia refleja una tendencia más amplia a minimizar o a marginar las consideraciones geográficas en la literatura, como si, para la toma de decisiones, las soluciones espaciales y los flujos fueran de importancia tangencial y no central. Si queremos empezar a entender cómo se ejerce el poder de manera iterativa, es necesario hacer una corrección. El espacio importa. Y para comprender qué tanto es relevante, se requiere de una investigación que revele y utilice materiales empíricos sobre los responsables de la toma de decisiones en el pasado de Sudáfrica a fin de crear nuevos conocimientos sobre el ejercicio del poder, las estructuras iterables en las que se interpretaron y negociaron las relaciones entre lo político y lo económico, y las maneras en las que la acumulación capitalista y su geografía conformaron 
las traducciones de los mundos prospectivos y los imaginarios que sobre éstos habrían querido crear los encargados de tomar decisiones.

Justamente, en este artículo se aborda este desafío intelectual mediante un análisis de la acción política durante los primeros años de la Unión Sudafricana, establecida en 1910. En este periodo, el hecho fundamental era el proyecto imperial británico en general, cuya administración estaba a cargo de numerosos servidores públicos británicos y funcionarios del gobierno que, en colaboración con la Oficina Colonial en Londres, fueron los responsables de la muy demandante tarea de navegar por una amplia gama de tensiones. Había, por ejemplo, divisiones entre los británicos y la población bóer herida, entre los intereses contradictorios que daban forma a las relaciones entre las casas mineras y los mineros, y en los debates y complicaciones que surgían de la llamada "cuestión nativa" del país. Si mantener el proyecto imperial en marcha exigía el desarrollo de una "fórmula imperial” (Ashforth, 1997, pp. 101-126), también significaba que los técnicos sobre el terreno tenían que hacer numerosas intervenciones con sus respectivos cálculos, destinados a alterar o a mantener constante la mezcla de materiales, intereses y movimientos de diversa índole. Generalmente, al igual que en las prácticas de gobernabilidad, la mayoría de estas acciones se ocupaban de episodios relativamente mundanos en los que la precedencia y la experiencia ofrecían orientación suficiente sobre qué se debía hacer.

Las cuestiones sensibles de importancia crítica, para las que se requerían decisiones que potencialmente alterarían la trayectoria, fueron menos comunes. De cualquier modo, como han argumentado los teóricos de la relacionalidad estatal y de los "efectos del Estado" en el periodo contemporáneo (Raco, 2003, pp. 75-95; Painter, 2006, pp. 752-774), el punto clave es que el imperialismo británico en la Sudáfrica de principios del siglo xx tuvo que ser administrado, manejado y masajeado. Quizá hubo diseños grandiosos, abstracciones de universalización e incluso algo que se aproximaba a una lógica imperial que servía de guía para orientar la actuación de los británicos, pero para tener una mirada de Estado (Scott, 1998) era necesario que los individuos convirtieran la autoridad en acción, mientras ima- 
ginaban, interpretaban y ejercían diariamente (y sobre el terreno) el poder iterativo.

Como tal, se sostiene que los materiales de archivo depositados por los funcionarios británicos de este periodo, en la Sudáfrica del siglo xx, of recen oportunidades para investigar y analizar cómo interactuaban los decretos cotidianos con el proyecto imperial. Dicho estudio puede sumarse a las contribuciones de los eruditos que han revelado la importancia de algunas de las personas más significativas durante esta época, sobre todo la de Alfred Milner, cuyas ideas y acciones, principalmente en las postrimerías de la guerra bóer, dieron forma al desarrollo de Sudáfrica de manera muy profunda (Le May, 1965; Marks y Trapido, 1979, pp. 50-80; Dubow, 1997, pp. 53-85). Milner era un alquimista de la fórmula imperial de Ashforth, un individuo que dejó una huella duradera en el lugar. Pensaba que Sudáfrica debía convertirse en un nuevo país donde las minas conservarían el acceso a la mano de obra migrante originaria de las reservas nativas y los colonos mantendrían para sí mismos las grandes extensiones de las mejores tierras agrícolas. Una Sudáfrica en la que los europeos asegurarían su dominación racial sobre los africanos, al mismo tiempo que lograrían un crecimiento económico que haría al país accesible y valioso para el capital industrial británico (Marks y Trapido, 1979). Las investigaciones detalladas sobre los muchos británicos que siguieron a Milner y que pretendieron dejar sus propias huellas (por cierto, menos innovadoras) sobre el proyecto imperial británico en el África del Sur del siglo xx no han aportado gran cosa. ${ }^{1}$ ¿Podría el análisis de las normas cotidianas arraigadas de los funcionarios británicos arrojar alguna luz sobre el proyecto imperial en Sudáfrica?

Un asunto de gran interés para dicha investigación es la manera en la que los actores británicos negociaron un proyecto imperial que comenzaba a derrumbarse. Sin duda, lo que estaba en juego, en retrospectiva, eran las ambiciones de Milner para el país, que no eran realistas. En primer lugar, era muy probable que los colonos que él creía que llegarían a Sudáfrica

${ }^{1}$ Antes de Milner pocos funcionarios habían sido objeto de investigaciones sostenidas. Una excepción es Theophilus Shepstone, por ejemplo, véase McClendon, 2010. 
siempre fueran a estar subordinados en relación con las minas, cuya demanda de mano de obra era tan enorme y creciente que incluso los gobiernos de la era del apartheid - con los extraordinarios poderes que adquirieron-lucharon para apoyar suficientemente al sector agrícola (Posel, 1991; O’Meara, 1996). El proyecto de una Sudáfrica propicia al asentamiento extensivo por parte de los europeos nunca tomó forma. Es más, las esperanzas de Milner de que los bóeres se contentarían con negociar con una industria minera que intentaba moldear el país a una imagen de su elección, no lograron anticiparse a la eventual fuerza del nacionalismo afrikáner, el cual encontró, entre las casas mineras, gran cantidad de evidencias que sugerían que los privilegios de los blancos estaban sobre terreno inestable. Finalmente, los errores en la visión de Milner fueron magnificados por los simpatizantes de un emergente "liberalismo colectivista” (véase Auld, 1975, pp. 78-101) en Gran Bretaña, quienes nunca vieron el proyecto imperial en Sudáfrica desde la misma óptica de Milner (o del gobierno conservador que lo envió al país en primer lugar) (Harris, 2010, p. 5). Sudáfrica, como el imperialismo, en general, nunca ocuparía las mentes de los políticos del Partido Liberal en Londres del modo en que Milner podría haber esperado de los futuros gobiernos.

Aunque las cuestiones importantes en Inglaterra y Europa eran sin duda las preocupaciones de los gobiernos liberales que habían sido electos en 1906 y 1910, el asunto sudafricano persistía. Los liberales lo abordaron, en parte, a través del Acta sobre Sudáfrica de 1909, surgida de las negociaciones con destacados políticos sudafricanos (Marks y Trapido, 1979, pp. 50-80), y que fue vista como un camino viable para llevar al país hacia adelante. Aspiraba a unificar las cuatro provincias -el Transvaal, Natal, el Estado Libre de Orange y la Colonia del Cabobajo un solo gobierno y a ubicarlas como dominio clave dentro del Imperio británico (Dubow, 1997, pp. 53-85). Sus poderes legislativos debían ser supervisados por Londres, con aportaciones sobre el terreno de un gobernador general. Sin embargo, ante la inminente formación de la Unión Sudafricana en 1910 y con una creciente preocupación por las intenciones militares de Alemania en Europa, el primer ministro Asquith optó por enviar como primer gobernador general a Herbert J. Gladstone 
(1854-1930), en lugar de Winston Churchill, quien era uno de los candidatos más capaces para ocupar el cargo.

\section{La Sudáfrica de Herbert Gladstone}

Gladstone había sido miembro del Parlamento desde 1880, jefe del Partido Liberal en la oposición (1899-1905) y secretario del Interior (1906-1910) en los gabinetes de Campbell-Bannerman y Asquith (Mallet, 1932). A diferencia de su padre, W. E. Gladstone, quien sin duda fue el político británico más importante del siglo XIX (Jenkins, 1995), Herbert había tenido una carrera mediocre. Sin embargo, como secretario del Interior - "un cargo más restringido que glorioso" (Jenkins, 1978, p. 175) - había adquirido una reputación de administrador relativamente competente y supervisó el desarrollo de reformas legislativas enfocadas a mejorar las condiciones de trabajo y de vida de las clases trabajadoras de Gran Bretaña (Mallet, 1932). Aunque no calificó como uno de sus muchos ministros talentosos (Jenkins, 1978, p. 175), fue miembro del gabinete de dos ambiciosos gobiernos liberales que introdujeron "reformas económicas, laborales, de bienestar social y 'desarrollo económico' sin precedencia en la historia británica” (Harris, 2010, p. 5).

En Sudáfrica, Gladstone ocupó una posición políticamente delicada, con muchas posibilidades de fracaso, pero fue al país con la esperanza de contribuir fructíferamente al desarrollo de mecanismos eficaces de gobernanza, guiado por su experiencia como secretario del Interior, que le daba una idea de lo que había que hacer (Mallet, 1932). A diferencia de Milner, en ese entonces Gladstone era simplemente un técnico de la fórmula imperial en Sudáfrica. Tenía poco poder para construir un cambio en el país. Al momento de su llegada, su posición respecto al gobierno de la Unión y los deberes que debía cumplir ya estaban prácticamente definidos.

Es justo suponer que la repercusión de Gladstone en la historia sudafricana fue menor. Quizá por eso es uno entre muchos funcionarios británicos cuyas contribuciones han quedado a la sombra de Milner. Sin embargo, Gladstone tuvo una oportunidad privilegiada de ver la conformación del país en una co- 
yuntura crucial y escribió sobre numerosos acontecimientos, por lo que en su archivo se encuentran análisis lúcidos y originales sobre la dinámica del país y sus tensiones emergentes. En el resto del artículo se aborda la ausencia de Gladstone en la literatura, pero también se investiga su interacción con el proyecto imperial, con la Sudáfrica que venía de la Guerra de los Bóeres, y su lugar como tomador de decisiones en un ejercicio iterativo del poder que lo obligó a negociar la geografía única de Sudáfrica. Se hace un análisis de sus respuestas escritas a un incidente crítico que ocurrió hacia el final de su estancia en el país. El examen de incidentes es una técnica ampliamente utilizada en las metodologías de investigación cualitativa debido a que centra la atención en las distintas respuestas e interpretaciones de los actores frente a la acción social en situaciones intensas en las que las contradicciones y las tensiones aparecen en primer plano (Tripp, 1994, pp. 65-76). Esta metodología general se aplica a propósito de la huelga de trabajadores blancos de los ferrocarriles y de las minas, en enero de 1914, situación tan grave para Gladstone que lo obligó a profundizar sobre el pasado, el presente y el futuro del país y a reflexionar por escrito. Primero se utilizan los textos de Gladstone acerca de estos eventos para presentar un relato descriptivo de su respuesta a la huelga. En seguida se exploran sus comentarios subsecuentes sobre las relaciones sociales en Sudáfrica, en su conjunto. Por último, en una discusión sobre los materiales revisados, se ofrece una interpretación crítica de la forma en la que Gladstone representó al proyecto imperial en Sudáfrica en el siglo xx y cómo interactuó con él.

\section{Gladstone y la huelga de enero de 1914}

La huelga de los trabajadores ferrocarrileros blancos en enero de 1914 y las acciones subsecuentes de los obreros de otros sectores - sobre todo de las minas de oro-culminaron en una huelga general (Beinart, 2001, p. 92) y provocaron circunstancias que, una vez más, pondrían a Gladstone en una posición incómoda. Sólo seis meses antes, en julio de 1913, en medio de una huelga de trabajadores mineros que provocó graves 
disturbios en el Rand y alrededor de él, había aceptado que el gobierno de la Unión utilizara 3000 soldados imperiales británicos para restablecer el orden público, tomar el control de la infraestructura más importante e intervenir las unidades habitacionales de las minas, donde se alojaban 200000 trabajadores mineros africanos (véase Hyslop, 2014, pp. 97-120). En Londres, algunos sectores de la prensa y ciertos miembros del Parlamento criticaron esta decisión, pero Gladstone se mantuvo firme y defendió con argumentos sólidos su resolución de apoyar el uso de las tropas imperiales.

Los acontecimientos de 1914 también podrían haber implicado el uso de tropas imperiales, pero no fue necesario. Cuando el gobierno de la Unión proclamó la Ley Marcial el 14 de enero, un día después de que estallara la huelga general, Gladstone tenía que decidir si acataría o no la proclamación del gobierno. En una carta enviada a Loulou Harcourt (Harcourt, 2006), secretario colonial en Londres, señaló: "la ultima ratio en forma de proclama que declarara un estado de Ley Marcial tendría que ser firmada por mí. No creo que, en estas circunstancias, tuviera alguna opción. Ciertamente no tengo ninguna duda" (Gladstone a Loulou Harcourt, 22 de enero de 1914, p. 405). Sin embargo, como en los acontecimientos de julio, Gladstone estaba dispuesto a defender su causa: "Si se ataca mi acción, estoy totalmente dispuesto a defenderla" (Gladstone a Harcourt, 22 de enero de 1914, p. 406).

Las circunstancias del Rand y del país en su conjunto le preocupaban, incluso si la situación general en ese momento era "muy diferente y mucho menos explosiva de lo que era en julio" (Gladstone a Harcourt, 14 de enero de 1914, p. 192). En parte, lo diferente fue el "tono de los líderes de los hombres en comparación con lo que era en julio [en la medida en que] están desalentando los actos de desorden" (Gladstone a Harcourt, 14 de enero de 1914, p. 192). Hubo "explosiones de dinamita en la línea ferroviaria" (Gladstone a Harcourt, 14 de enero de 1914, p. 193), escribió en un envío posterior y puso énfasis en que:

un tren correo que salió de Johannesburgo en la noche del 9 de enero con dirección a Ciudad del Cabo estuvo dentro del margen de destruc- 
ción a una distancia de aproximadamente cuatro millas de Johannesburgo. Alguien había colocado dinamita en la línea, y cuando el tren pasaba por ahí a cierta velocidad, una sección de la rueda del motor principal, y del riel, explotaron. Afortunadamente, aunque el tren tuvo que parar durante la noche para ser reparado, conservó los metales (Gladstone a Harcourt, 22 de enero de 1914, p. 176).

Sin embargo, se trataba de acciones aisladas, contrarrestadas por el hecho de que "se han organizado patrullas de huelguistas para preservar el orden" (Gladstone a Harcourt, 22 de enero de 1914, p. 192).

Otra diferencia "singular y esclarecedora" (Gladstone a Harcourt, 22 de enero de 1914, p. 191) entre la huelga de 1914 y los acontecimientos de 1913 tuvo relación con el nivel de preparación del gobierno de la Unión. En julio de 1913, él le había sugerido a Harcourt que:

Después de lo que pasó era fácil culpar al gobierno por su falta de previsión. La organización del Departamento de Gobierno era inmadura, en su mayoría, los ministros mismos no tenían experiencia en la administración y se vieron obstaculizados por las disensiones partidistas. Y más allá de todas estas consideraciones que pueden ser instadas en su defensa, Sudáfrica casi no había tenido oportunidades para estudiar los complicados problemas que surgen de las relaciones entre patrones y empleados en las grandes industrias (Gladstone a Harcourt, 22 de enero de 1914, p. 187). ${ }^{2}$

En resumen, Gladstone llegó a la conclusión de que la huelga de julio de 1913 había expuesto un aparato estatal mal preparado para hacer frente a un desafío tan importante a la autoridad. El gobierno era joven y carecía de la suficiente comprensión sobre la manera en la que podría intervenir eficazmene en la sociedad. Uno de los objetivos de Gladstone era alentar a este joven gobierno a reforzar sus poderes y a ampliar su capacidad de acción.

Por lo tanto, estaba feliz de que para enero de 1914 el aparato del Estado hubiera mejorado. Ahora el gobierno podía confiar en las Fuerzas de la Defensa reglamentadas por el Acta sobre la Defensa No. 13 de 1912 y pedirles que se trasladaran

${ }^{2}$ Fue muy fácil llegar a esas conclusiones debido a que, como destaca Hyslop (2014, p. 98), “el Estado sudafricano era visiblemente frágil”. 
a las áreas del campo afectadas. Para el "sábado 10 de enero, las Fuerzas de la Defensa, como tales, se habían reunido en número suficiente para hacer frente a cualquier tipo de disturbio que pudiera surgir" (Gladstone a Harcourt, 22 de enero de 1914, p. 192; Hyslop, 2014, p. 116). ${ }^{3}$ La llegada de estas fuerzas provenientes de las zonas rurales había sido "la característica más pintoresca de la situación, y también, probablemente, un factor decisivo para prevenir desórdenes graves" (Gladstone a Harcourt, 15 de enero de 1914, p. 228). La movilización de las Fuerzas de la Defensa por parte del gobierno había sido realizada...

admirablemente. Parecía que por todos lados los comandos bóeres estaban disponibles. Cada vez había más trenes funcionando. Los hombres armados mantenían bajo su control todos los centros ferroviarios importantes y patrullaban las líneas. Había aproximadamente 15000 en Johannesburgo y en el Arrecife, o se encontraban a corta distancia (Gladstone a Harcourt, 14 de enero de 1914, p. 191).

Cuando la Ley Marcial fue proclamada, Gladstone concluyó que "de hecho, el gobierno tiene la situación bajo control. En cada etapa se ha anticipado a los huelguistas" (Gladstone a Harcourt, 14 de enero de 1914, p. 192). Por ejemplo, "cuando aparecieron los piquetes de hombres [...] descubrieron que las estaciones [de tren] y todos los centros importantes o los puntos vulnerables ya estaban ocupados por hombres armados" (Gladstone a Harcourt, 14 de enero de 1914, p. 192). Por lo tanto, elogió al gobierno de la Unión. Lejos de que las acciones revelaran un Estado débil, le dijo a Harcourt:

Dudo que pueda encontrarse algún precedente sobre medidas de protección tan completas, rápidas y eficaces. Aunque el área problemática es mucho mayor de lo que era en julio, no es probable que en la situación actual existan los peligros a los que nos enfrentábamos entonces. La situación actual muestra a un gobierno abrumadoramente fuerte (Gladstone a Harcourt, 14 de enero de 1914, p. 193; Hyslop, 2014, pp. 116-119). ${ }^{4}$

${ }^{3}$ Hyslop (2014) destaca que: "En la represión de los sindicalistas de Rand se hizo un uso particularmente intenso de los bóeres del campo. Vistos desde fuera, parecía que los aspectos militares de la operación de enero de 1914 iban bien para el gobierno”.

${ }^{4}$ Para Hyslop (2014), el gobierno había controlado la situación, pero la huelga de enero llevó la atención hacia las tensiones entre los bóeres del campo y el go- 
Todos los acontecimientos antes mencionados significaban que, esta vez, era poco probable que el gobierno requiriera del apoyo de las tropas imperiales, a menos que "fuera deseable utilizarlas para resguardar al Protectorado y a los indígenas portugueses, ya fuera en los complejos habitacionales o con una finalidad de repatriación [a sus regiones o países de origen] si desafortunadamente dicha medida tuviera que ser adoptada" (Gladstone a Harcourt, 14 de enero de 1914, p. 194). Sin embargo, la repatriación —una medida dramática y complicada- sólo sería necesaria "si las circunstancias exigieran, en última instancia, el cierre completo de las minas por un largo periodo. No obstante, se espera que tal emergencia no suceda" (Gladstone a Harcourt, 15 de enero de 1914, p. 234).

Mientras tanto, estaba impresionado:

se han tomado medidas complejas para la protección de los nativos en las minas y para la prevención de disturbios entre ellos. Se mantiene en reserva una gran provisión de víveres y se dice que son suficientes para abastecer el racionamiento durante un mes; además, los complejos habitacionales se encuentran debidamente custodiados (Gladstone a Harcourt, 15 de enero de 1914, p. 233).

Por consiguiente, Gladstone identificó entre el público en general "una completa ausencia de pánico" y un sentido de "plena confianza en la efectividad de las medidas cautelares adoptadas por el gobierno" (Gladstone a Harcourt, 15 de enero de 1914, p. 233). "Se tomaron medidas enérgicas", escribió a Harcourt el 22 de enero, "y con tal prontitud, que en cada punto los huelguistas se encontraron rebasados e impedidos para actuar" (Gladstone a Harcourt, 22 de enero de 1914, p. 177).

\section{Las perspectivas de Sudáfrica y la cuestión racial y de clase}

La huelga de enero de 1914 fue sin duda un incidente de gran importancia durante la estancia de Gladstone en Sudáfrica. Una

bierno, mismas que se encendieron en agosto de 1914 cuando comenzó la guerra con Alemania. 
vez que la huelga fracasó, en parte bajo el peso severo de la Ley Marcial, las responsabilidades de Gladstone le exigieron desarrollar y enviar a Londres alguna justificación de sus acciones. Al hacerlo, por supuesto, se vio obligado a hablar de lo que él creía que era la gravedad de la situación: le habló a Harcourt del potencial de "perjuicio" por parte de los trabajadores en huelga y del riesgo de que los "nativos se salieran de control" (Gladstone a Harcourt, 22 de enero de 1914, p. 167). Pero también fue más allá de informar sobre la situación inmediata y presentó un análisis breve, pero esclarecedor, de las tensiones más profundas que habían conformado los acontecimientos de julio de 1913 y de enero de 1914. En esta parte del artículo me enfocaré en los argumentos de Gladstone.

En un despacho anterior enviado a Harcourt, Gladstone se refirió a los acontecimientos de julio de 1913 y observó que se avecinaban serios problemas; "su estallido es solamente una cuestión de tiempo" debido a ...

la total ausencia de una relación correcta entre los dueños de las minas y los mineros, misma que debería existir en una industria organizada; la creciente amargura de una gran parte de los hombres; el deseo de [los dueños de las minas] de controlar y de tener influencia para orientar el rumbo de los hechos; el feroz resentimiento [de los mineros] por la insensibilidad demostrada en épocas pasadas por las casas mineras, ya que no hicieron nada para hacer frente a los estragos de la tisis, y lo inadecuado de los remedios que aplicaron cuando finalmente el Parlamento fue obligado a atender el asunto... (Gladstone a Harcourt, 14 de enero de 1914, p. 187).

En enero de 1914 sugirió que los asuntos en cuestión eran problemas de la misma índole. Haciendo un esbozo del contraste con Gran Bretaña, donde durante su mandato como ministro del Interior había contribuido a introducir una legislación para alterar las relaciones generales de producción, en un despacho enviado a finales de enero, Gladstone enfatizó la importancia de desarrollar una determinada estructura de relaciones de trabajo entre patrones y empleados:

En todos los grandes empleos en el Reino Unido se encuentra un gran porcentaje de hombres que han trabajado toda su vida, y de muchos que han trabajado durante largos años para un solo patrón. Las buenas 
condiciones de trabajo mantienen a los hombres contentos y leales, y aunque puede ser que participen en huelgas, esto, por regla general, no significa una ruptura personal con los propietarios y los gerentes (Gladstone a Harcourt, 31 de enero de 1914, p. 190).

Por lo tanto, Gladstone estaba convencido de que en Gran Bretaña la estabilidad y la permanencia habían producido "hombres contentos y leales". Y, desde su punto de vista, el desarrollo de la situación en Sudáfrica no se acercaba a lo anteriormente descrito. "En el Arrecife hay buenos patrones", le dijo a Harcourt, "y hay buenos hombres que trabajan con ellos de manera continua”. Pero, continuó: "los administradores varían, y los grupos mineros varían. Las afinidades personales entre los patrones y los empleados son casi inexistentes" (Gladstone a Harcourt, 31 de enero de 1914, p. 190). Lo que estaba en duda eran las prácticas profundamente arraigadas en el lugar de trabajo en Sudáfrica:

En lo que respecta a los seres humanos, la gestión, independientemente de sus capacidades de trabajo, se ha dejado principalmente al azar. La práctica de presentar una notificación con 24 horas de anticipación por ambas partes para dar por terminada la relación laboral se ha convertido en algo fundamental. Sin embargo, resulta prácticamente fatal para la lealtad y el entendimiento mutuos. Los hombres, sabiendo que pueden conseguir un trabajo tan bueno o mejor en otro lugar, continuamente van de una mina a otra. Del mismo modo, los gerentes no tienen ningún impedimento para realizar despidos sumarios, aun sabiendo que un hombre, que tal vez tiene una familia que mantener, se quedará sin trabajo. Los mineros, por lo tanto, son unidades independientes. Esto quizá pueda tener sus ventajas; sin embargo, la independencia mutua característica de los patrones mineros y de los empleados en el arrecife impide el crecimiento del respeto y la consideración mutuos, lo cual es esencial para establecer condiciones de trabajo sanas y permanentes (Gladstone a Harcourt, 31 de enero de 1914, p. 191). ${ }^{5}$

Como tal, una cuestión clave surgida de la "dinámica de la posición industrial y social general en Sudáfrica” (Gladstone a Harcourt, 31 de enero de 1914, p. 191) fue la falta de estabilidad; un cierto grado de impermanencia de flujo. Al comentar

${ }^{5}$ Hyslop (2014, p. 99) destaca que "los autocráticos dueños de las minas" habían "bloqueado los intentos de ampliar la base de la economía industrial" y que, una década antes, incluso habían experimentado importando trabajadores chinos. 
sobre la situación de los mineros, por ejemplo, subrayó que "muchos de ellos no están casados, se cambian de una mina a otra y el número de quienes entre ellos tienen la intención de establecerse en el país es mucho menor que en el caso de los ferrocarrileros, quienes lo hacen esperando obtener pensiones. Como clase, los mineros son más inquietos y menos previsores" (Gladstone a Harcourt, 31 de enero de 1914, p. 189).

De manera aislada, tales niveles de impermanencia quizá no hubieran importado. Pero Gladstone había notado en Sudáfrica no sólo la situación industrial, sino también la manera en la que se relacionaba con las perspectivas materiales más amplias del país. En muchos aspectos, los intereses de los mineros itinerantes se enfrentaron a los intereses de quienes, según Gladstone, dependía la "prosperidad última y, en gran medida, la prosperidad actual del país" (Gladstone a Harcourt, 22 de enero de 1914, p. 174), es decir, los campesinos blancos. Lo que tanto parecía frustrarlo sobre la manera en la que habían actuado los trabajadores blancos - ferrocarrileros y mineros-, era precisamente que los intereses de los agricultores del país habían sido dejados de lado:

En julio, al igual que en enero, los líderes sindicalistas parecieron ignorar las opiniones de todas las clases, exceptuando las de las multitudes que se reunieron a escuchar sus discursos [...] Los 97000 agricultores, que son la columna vertebral industrial de África del Sur, nunca fueron mencionados. Dispersos como estaban a lo largo de una gran superficie de terreno, parece que pensaron que podían ser ignorados por el Trades Hall. No son capitalistas. Y como clase no ven con simpatía a los dueños de las minas. Ciertamente no eran hostiles a las exigencias razonables de los obreros blancos. Estos hombres están orgullosos de su país y odian el desorden. Esperan que el gobierno atienda sus intereses y les otorgue protección. Constituyen la masa de la población. Como están establecidos en esas tierras, sus intereses son total y permanentemente sudafricanos. Por lo general, los agricultores no tenían ningún empacho en ver con desaprobación y hostilidad los intentos de un pequeño número de individuos sin ningún interés permanente en el pais, para subvertir al gobierno (Gladstone a Harcourt, 31 de enero de 1914, p. 195; el énfasis es mío).

Por lo tanto, al observar a los agricultores, Gladstone veía en ellos a los potenciales constructores de la nación sudafricana. Mientras que los mineros no tenían ningún "interés 
permanente" en el país, Gladstone sostenía que las esperanzas del gobierno de la Unión de crear un dominio fuerte dentro del imperio dependían de los colonos, quienes tenían intereses "total y permanentemente" sudafricanos. Los agricultores y los colonos, no los mineros, eran quienes serían necesarios para establecer una sociedad sudafricana adecuada.

Lo que resultaba crucial era que Gladstone no era tímido para establecer la conexión entre la raza, que era el factor que obviamente complicaba las relaciones industriales en Sudáfrica, y su crítica hacia los mineros. ${ }^{6}$ Sugirió que la razón principal de que "las condiciones de empleo y salarios en Sudáfrica difirieran materialmente de las que prevalecían en el Reino Unido" (Gladstone a Harcourt, 31 de enero de 1914, p. 187) era que los trabajadores blancos de las minas tenían la intención de mantener sus privilegios raciales sobre los africanos en el lugar de trabajo. Los trabajadores blancos se apoyaban fuertemente en una arquitectura legal que "prohíbe a un nativo desarrollar un trabajo calificado; no porque no pueda hacerlo tan bien, sino para mantener el monopolio del trabajo calificado en manos europeas con una tasa de salario que sea establecida por ellos mismos" (Gladstone a Harcourt, 31 de enero de 1914, p. 187). Esto no le sorprendía: "Los trabajadores blancos de Sudáfrica son en realidad una clase media privilegiada" (Gladstone a Harcourt, 31 de enero de 1914, p. 187) y se encuentran entre los "mineros mejor pagados del mundo" (Gladstone a Harcourt, 31 de enero de 1914, p. 190). Mientras que los mineros blancos ganaban $£ 20$ (libras esterlinas) y hasta $£ 40$ al mes, los trabajadores africanos ganaban solo $£ 3$ o $£ 4$. Pero "la mayor parte del trabajo pesado y demandante del país es realizado por los nativos" (Gladstone a Harcourt, 31 de enero de 1914, p. 187).

Delineando de nuevo los contrastes con Gran Bretaña, hizo la siguiente evaluación de una parte clave de la escena política en Sudáfrica:

${ }^{6}$ Esto no significa que desestimara por completo las legítimas quejas de los mineros. Simpatizaba con su situación, especialmente "por el terrible tributo de vida exigido por la tisis, [la cual] amarga y estrecha su punto de vista". El "espíritu feroz e intransigente entre los hombres que están propensos a contraer la tisis —señalópuede ser entendido fácilmente". "Una vez que la enfermedad los atrapa, [sus] elevados salarios no les sirven de nada” (Gladstone a Harcourt, 31 de enero de 1914, p. 190). 
Si el Partido Laborista Blanco pretende ocupar la posición del Partido Laborista en nuestro país y tener el mismo derecho a la simpatía del pueblo, la respuesta es que el Partido Laborista en Gran Bretaña incluye a una gran masa de trabajadores no calificados que devengan salarios que suelen ser terriblemente bajos. El partido laborista aquí excluye a los hombres no calificados, no porque su trabajo sea deficiente, sino porque su piel es negra, ese trabajador está fuera del ring, es un hombre, pero no un camarada (Gladstone a Harcourt, 31 de enero de 1914, p. 188).

En consecuencia, sugirió que "mientras los obreros blancos mantengan esta posición, es obvio que sus ataques en contra de sus patrones y de las 'clases capitalistas', en gran medida, se reviertan sobre ellos mismos. El derecho que ellos reclaman para sí con relación a vender su trabajo al mejor precio que puedan obtener, lo niegan al nativo" (Gladstone a Harcourt, 31 de enero de 1914, p. 188). El africano, dijo, "se estaba esforzando a su manera para mejorarse a sí mismo”, pero la estructura de las relaciones industriales, en última instancia, implica que "no tiene permiso de vender su trabajo y sus competencias, excepto que lo haga por una cifra que esté muy por debajo de su valor económico, a fin de que el alto salario del hombre blanco pueda seguir siendo más alto; sin importar que su capacidad y energía justifiquen o no dicha paga" (Gladstone a Harcourt, 31 de enero de 1914, p. 188).

En cierto nivel, los puntos de vista de Gladstone en relación con el trabajador blanco eran simplemente un reflejo de su profundo liberalismo: los trabajadores blancos estaban presentando obstáculos innecesarios en el camino del tipo de desarrollo económico que él conceptualizaba como progreso. Pero, en otro nivel, es posible discernir una comprensión relativamente astuta de lo que, desde su punto de vista liberal, eran los riesgos que enfrentaba el país. La dinámica social e industrial en Sudáfrica se basaba en una ingeniería peculiar de explotación de clase y dominación racial (Ashforth, 1997; Marks y Trapido, 1979; Hyslop, 2014). La explotación de los africanos no le importaba a Gladstone; de hecho, su punto de vista era que la explotación era para su propio bien, algo que ayudaba al africano en su esfuerzo por mejorarse a sí mismo. Sin embargo, se sentía indudablemente perturbado por ciertas formas evidentes de dominación racial. Las actitudes hacia 
los africanos entre algunos europeos lo frustraban. Además, si ya había una "cuestión racial [que] impregnaba a toda Sudáfrica, independientemente de las fronteras" (borrador mecanografiado de la autobiografía inédita de Gladstone, p. 202), ésta se complicaba con los blancos pobres (sobre la política más amplia del "blanquismo" [whiteism] pobre, véase Bottomley, 2016, pp. 76-86). En una carta personal y confidencial que le escribió a Harcourt en julio de 1911 le había dicho que los blancos pobres eran "o bien consumidores, u hombres sin ningún tipo de capacitación que sólo eran aptos para el trabajo manual no calificado". Destacó que:

Con demasiada frecuencia, resulta repugnante ver a los blancos calificados a cargo de una cuadrilla de nativos. Todo el día haraganean, sólo dirigen y se imponen. Con las manos en los bolsillos, la pipa en la boca y no pocas veces hinchados de tanto beber. Si esto continúa, la mano de obra blanca se va a hundir (Gladstone a Harcourt, 14 de julio de 1911, p. 187).

Criticaba que los blancos pobres no trabajaran con los africanos y tenía una idea de cuáles serían las consecuencias económicas: "Si [trabajaran con los africanos], ¿cómo se puede proporcionar un salario digno a un hombre blanco frente al trabajo más barato e igualmente bueno, o incluso mejor, del nativo? Éste es el quid de la situación" (Gladstone a Harcourt, 14 de julio de 1911, p. 187).

Al igual que su crítica sobre la barrera de color en las minas, Gladstone encontró poco que admirar en una sociedad sudafricana que pretendía "evitar el progreso de los nativos con la falsa ilusión de que eso les daría trabajo a los blancos pobres" (Gladstone a Harcourt, 14 de julio de 1911, p. 187). En lugar de socorrer a los blancos pobres a costa de la discriminación en contra de los africanos, quienes según él cada vez más "estaban adquiriendo rápidamente mayores destrezas y hábitos de trabajo más estables" (Gladstone a Harcourt, 14 de julio de 1911, p. 187), Gladstone expresó su esperanza de que el país viera el desarrollo de "una política blanca que fuera más sensata sobre la cuestión nativa" (Gladstone a Harcourt, 14 de julio de 1911, p. 188). Sudáfrica, adujo Gladstone, "necesita toda la mano de obra que pueda obtener y la política correcta es desarrollar las 
capacidades nativas. El hombre blanco debe competir o tendrá que irse. Y en muchas partes de Sudáfrica puede competir. La expansión industrial seguirá el desarrollo más completo de las capacidades nativas y eso aumentará las oportunidades para el hombre blanco".

\section{Discusión}

Las secciones precedentes describen las interacciones y las acciones de Gladstone relacionadas con la huelga de 1914. A diferencia de otros funcionarios británicos que pasaron algún tiempo en Sudáfrica, hombres como Milner especialmente, es justo concluir que Gladstone sólo desempeñó una pequeña parte en el trasfondo del drama que se desarrollaba en ese país. Pero mientras se encontraba en el escenario durante su mandato como gobernador general, particularmente en el contexto del incidente crítico en el que me he centrado, presentó una gama de ideas liberales sobre distintas maneras en las que el país podría haber mejorado sus perspectivas de futuro. ¿A qué conclusiones podríamos llegar a partir de sus comentarios?

Se consideran dos cuestiones: la primera se refiere al poder iterativo, el cual sale a la luz al analizar las propuestas liberales de Gladstone. Como he descrito, expresó su preocupación por lo que iba mal en Sudáfrica y formuló propuestas sobre cómo el país podría desarrollarse de manera más estable. Quería que hubiera un orden liberal en las minas: relaciones más estables entre los dueños de las mismas y sus trabajadores (blancos); un mejor entendimiento mutuo sobre sus intereses compartidos. Entonces les pidió a los blancos que adoptaran una actitud más progresista hacia los africanos, para que surgiera una sociedad más liberal en la que los blancos pobres no tuvieran prioridad sobre una población africana cada vez más capaz. Gladstone tenía razón al ver las conexiones entre estas dos cuestiones: en Sudáfrica, las relaciones de producción en las minas habían sido, y seguirían siendo, instrumentales, y en ningún momento era posible comprender la minería (o la vida social en general) sin captar la importancia de las singulares estructuras que conformaban las relaciones raciales del país. El capitalismo, por un 
lado, y las nociones liberales sobre el progreso, por el otro: ambos estaban ligados a la política de clase y raza en Sudáfrica. Sin embargo, debe quedar claro que el liberalismo de Gladstone era incapaz de aceptar la profundidad y la intensidad de las contradicciones que definían la manera en la que Sudáfrica estaba tomando forma. Vio las conexiones entre clase y raza y creía en las virtudes del orden liberal como una manera de transformar adecuadamente la sociedad, pero subestimó hasta qué punto la acumulación en Sudáfrica requeriría de la persistencia de las prácticas no liberales.

En cuanto a las minas, por ejemplo, su idea de que a la minería le vendría mejor establecer relaciones más estables con los mineros provenía de la experiencia británica; un lugar donde la minería del carbón se había desarrollado en comunidades cerradas y profundamente patriarcales que era necesario reproducir en la familia minera. Como Doreen Massey ha señalado:

El trabajo de un minero tenía como consecuencia enormes cargas domésticas para su esposa y su familia. El trabajo subterráneo era sucio [...] La ropa de trabajo tenía que ser hervida en ollas de cobre sobre un fuego que debía calentar toda el agua para lavar la ropa, lavar los pisos y para que la gente se bañara (Massey, 1994, p. 193).

Pero la minería en Sudáfrica era una entidad completamente diferente. En primer lugar, las "reservas de oro del país eran enormes, pero el contenido de oro de los llamados arrecifes era escaso y éstos se encontraban a una profundidad considerable" (Cox, 2016, pp. 10-26). Éste era un problema que la industria minera negociaba al instituir el sistema retrógrado de mano de obra migrante. Por otra parte, la industria minera de Sudáfrica dependía intensamente de gerentes e ingenieros blancos calificados, pero itinerantes, muchos de los cuales sólo buscaban ganar altos salarios mientras trabajaban en el país durante un corto tiempo (Tuffnell, 2015, pp. 53-76; Hyslop, 2014). Gladstone no hizo ninguna crítica sobre el sistema de mano de obra migrante, pero ciertamente expresó la esperanza de que pudiera surgir una situación más permanente en relación con los trabajadores blancos. Deseaba condiciones más estables en contraste con el flujo que encontró en la industria minera. Pero las contradicciones de la acumulación excluyeron esta posi- 
bilidad: como Gladstone señaló, los mineros blancos calificados ambulantes ya eran caros, lo cual significaba que ofrecerles condiciones más seguras sólo aumentaría los costos y amenazaría la rentabilidad en la industria. Por otra parte, en esta etapa, la industria ya estaba interesada en capacitar a los africanos para realizar tareas más calificadas (Callinicos, 1985). Si los dueños de las minas hubieran podido hacerlo, habrían africanizado toda la fuerza de trabajo. Los trabajadores blancos sabían esto, de ahí sus esfuerzos para instituir la reservación de puestos de trabajo [para los blancos] y el afán por parte de las minas para prevenir dicha africanización: un debate que llegó a un punto crítico con la revuelta del Rand en 1922 y que pasó a convertirse en una característica central de la estructura del apartheid (Posel, 1991). El capital era mucho más daltónico de lo que los trabajadores blancos podían tolerar. En resumen, el tipo de liberalismo promovido por Gladstone podría haber sido "comprado" en las minas británicas, pero no en Sudáfrica, donde la presión de las contradicciones del capitalismo obligó a la industria minera a adoptar un acuerdo espacial profundamente antiliberal entre las fuerzas; un acuerdo que estimulaba un mayor florecimiento de los talentos africanos, pero sólo porque satisfacía la necesidad de la industria para obtener beneficios. No hay nada en los archivos de Gladstone que sugiera que él comprendía esta dinámica.

Si las opiniones liberales de Gladstone sobre las minas subestimaban la medida en la que la acumulación en Sudáfrica requeriría la implantación de prácticas intransigentes, sus puntos de vista sobre la demografía futura ideal de Sudáfrica también reflejaban un mal cálculo sobre lo que tenía frente a él. Al igual que Milner, sus esperanzas se centraban en el surgimiento de un país en cuyas tierras se estableciera un mayor número de blancos. Su fe estaba en el colono blanco resuelto, con un interés "permanente" en el país, con una nueva ciudadanía emergiendo bajo el gobierno de la Unión, una ciudadanía blanca "total y permanentemente sudafricana". Para Gladstone, entonces, el "arreglo espacial” (Harvey, 1982) de las tensiones en Sudáfrica -desbordadas en julio de 1913 y de nuevo en enero de 1914- consistía en atraer a más colonos, precisamente porque una población con una apuesta tal en el futuro del país en- 
gendraría las condiciones adecuadas para establecer un orden liberal: después de todo, eran los blancos de las áreas rurales quienes habían acudido al llamado del gobierno en enero de 1914, y él vio en ese movimiento la evidencia del surgimiento de una nueva conciencia nacional. Para Gladstone era posible desarrollar una sociedad liberal a partir de las bases sociales, políticas y culturales que los colonizadores blancos habían construido para proteger sus intereses en el país a largo plazo. Sin embargo, aquí Gladstone, de nuevo, parece haber malinterpretado lo que se estaba conformando en Sudáfrica. Por un lado, había que considerar el poder de la industria minera del país y su enorme demanda de mano de obra, un factor que los agricultores blancos tendrían que impugnar en las siguientes décadas: en efecto, la estructura geográfica de la economía sudafricana establecía un techo sobre las ambiciones que tenía de convertirse en una sociedad que tuviera como base a los colonos asentados en su territorio (O’Meara, 1996). Por otra parte, incluso si Sudáfrica hubiera tenido más colonos, dicha sociedad presuponía prácticas retrógradas, como aprobar leyes para preservar el acceso a la mano de obra africana o leyes discriminatorias como la Natives Land Act [Acta sobre la Tierra de los Nativos], de 1913, para apuntalar el sistema de mano de obra migrante y dejar de lado la tierra para que los blancos fueran sus únicos dueños. Su clamor para incrementar el número de colonos blancos (para asegurar que el control europeo sobre la tierra se afianzara y se expandiera) exigía que las prácticas intolerantes en Sudáfrica, en lugar de reducirse, simplemente se volvieran más extensivas. La mezcla ideal de Gladstone de aumentar el número de colonos para obtener una solución espacial más estable nunca fue una receta para el liberalismo.

Sin embargo, las esperanzas de Gladstone no eran simplemente que los colonos lograran y alentaran la estabilidad que él imaginaba era necesaria para que emergiera el orden liberal. Por el contrario, tenía la firme convicción de que los blancos necesitaban superar la "falsa ilusión” que tenían respecto a sus relaciones con los africanos. Quería que hubiese "una política blanca más sabia”, una política liberalizadora: no sólo una nueva política colectiva sobre el lugar de trabajo para desalentar la impermanencia y el flujo, sino también para desmantelar la ba- 
rrera del color y poner fin a las políticas discriminatorias que impedían el desarrollo de los trabajadores con base en el color de su piel. El proyecto imperial, tal como lo imaginaba, necesitaba reformas liberales. Por lo tanto, en un discurso de despedida que pronunció en Ciudad del Cabo en 1914, habló de la "gran dificultad de Sudáfrica en relación con el color" (Discurso de Gladstone en Ciudad del Cabo, 10 de julio de 1914, p. 307); comentó que esta "pequeña población blanca que en gran medida se encuentra dispersa en un gran espacio de tierra agrupada en pequeñas comunidades [necesitaría desarrollar una] fuerza moral lo suficientemente fuerte como para aplastar las fuerzas de la ignorancia y los prejuicios" (Discurso de Gladstone en Ciudad del Cabo, 10 de julio de 1914, p. 308). Y advirtió a las élites de Sudáfrica sobre la necesidad de encontrar una solución más liberal:

Sólo puedo expresar mi convicción de que si la opinión pública es apática y la cuestión del color es dejada a sus posibilidades políticas, la autoridad moral del hombre blanco continuará disminuyendo, los hombres de color perderán la confianza que puedan tener, cada vez más los nativos tomarán lo peor de los hombres blancos y, al final, no tendrán nada sobre que construir, excepto la fuerza (Discurso de Gladstone en Ciudad del Cabo, 10 de julio de 1914, p. 307; el énfasis es mío). ${ }^{?}$

Gladstone realizó, entonces, el cierre de sus funciones como gobernador general en Ciudad del Cabo. Había sido el protagonista del proyecto imperial: desempeñó un papel relativamente menor en la historia de Sudáfrica y, por lo tanto, en el papel que Gran Bretaña jugó en ella. Pero si tomamos como base su interpretación de las tensiones expresadas en la huelga de 1914, se puede afirmar que Gladstone también comprobó las limitaciones de ese proyecto. Vio la necesidad de cambio, de reformas liberales e, incluso, de comenzar a conceder terreno a los pueblos subordinados del país. Gladstone estaba cuestionando las bases del dominio británico y blanco en Sudáfrica. Su experiencia en las huelgas le había dado razones para mirar el país, imaginar cómo podría surgir en el futuro y proponer reacomodos de las relaciones socioespaciales, materiales y de

${ }^{7}$ La fuerza, por supuesto, ya era la base para mantener la dominación racial en Sudáfrica. Véase Evans, 2005, pp. 183-202; Graham, 2012; Higginson, 2001, pp. 95-124. 
los flujos. Imaginó la manera en la que el proyecto imperial podría desplegarse de modo diferente a través de estos nuevos ajustes. Como ya se señaló, no era posible que las ideas de Gladstone propiciaran la construcción de la sociedad liberal en la que él parecía creer. Tal vez lo sabía. De hecho, quizá justamente de acuerdo con las expectativas que Butler quiere que tengamos, Gladstone practicaba una forma de poder iterativo: una habilidad y propensión a actuar que emerge de un acto performativo de ida y vuelta [recíproco/de doble vía], que involucra realidades empíricas sobre el terreno (influido por la dinámica de la acumulación). Pero, en el caso de Gladstone, su poder iterativo también reflejaba un compromiso continuo con la racionalidad imperial y su experiencia de traducir los intereses de Gran Bretaña en Sudáfrica a través del prisma de sus ideas y preceptos liberales. Sus ideas sobre las reformas liberales expresaron este poder iterativo y produjeron propuestas defectuosas para reorganizar la geografía única del país. Sin embargo, independientemente de que estas propuestas fueran o no erróneas, el poder iterativo de Gladstone, como tomador de decisiones dentro de una estructura iterable más amplia, había sido claramente desplegado durante sus últimos meses en el país.

Esto me lleva al segundo tema por discutir. El discurso de Gladstone en Ciudad del Cabo mostró su optimismo sobre el futuro de Sudáfrica. Él estaba, dijo, "lleno de esperanzas" (Discurso de Gladstone en Ciudad del Cabo, 10 de julio de 1914, p. 308) en relación con el país. Gladstone mantuvo dicho optimismo sin pensar en absoluto en la necesidad de una Sudáfrica liberal para crear igualdad racial, una franquicia no racial y, por lo tanto, un gobierno de mayoría. Sus esperanzas de estabilidad estaban fuera de lugar mientras se negara a reconocer las prácticas antiliberales incrustadas en la fallida aventura colonial-imperial en Sudáfrica: una aventura que dependía de una forma de acumulación capitalista y un proceso de construcción del Estado estrechamente asociados a sus núcleos, y que sólo lograría sobrevivir desarrollando tecnologías disciplinarias violentas, por ejemplo a través de leyes de paso más sofisticadas, formas de gobernanza urbana definidas más intensamente por la segregación y numerosas leyes diseñadas para suprimir a la oposición (Posel, 1991; O’Meara, 1996; Platzky y Walker, 
1985). Precisamente debido a que su "interés permanente" en el país dependía tanto de las estructuras racializadas para la explotación de la mano de obra africana, los europeos se vieron obligados a construir formas violentas de gobernanza. Por lo tanto, al final, el mayor defecto del liberalismo de Gladstone fue su negativa a entender a los pueblos subordinados de $\mathrm{Su}$ dáfrica. Los africanos, por ejemplo, ciertamente tenían un "interés permanente" en el país, pero, según Gladstone, su interés también era reducido porque eran negros $y$, en última instancia, estaba fuera de lugar. Así, aunque durante su mandato como gobernador general Gladstone pudo haber investigado algunos de los límites del imperialismo, en el centro de su interacción con Sudáfrica había una incapacidad para evitar cometer el error colonial clásico en África: subestimar y, en última instancia, malentender la contribución que los africanos podrían haber hecho (y estaban destinados a hacer) a la vida social, económica y política del país. Los zulúes, por ejemplo, eran, entre otras cosas, "muy chistosos", "muy interesantes" e incluso "magníficos compañeros" (borrador mecanografiado de la autobiografía inédita de Gladstone, p. 318). De manera elocuente, para Gladstone los africanos eran “de cierta manera, niños” (borrador mecanografiado de la autobiografía inédita de Gladstone, p. 317) y, como tales, no podía ver la necesidad de que imaginaran futuros más brillantes que depender de los europeos. La noción de Gladstone del africano como niño -incivilizado, subdesarrollado e inútil sin la tutoría de los europeos-era típica de la mentalidad colonial británica (Mamdani, 1996).

Sin embargo, durante su estancia en el país surgió una incipiente sociedad civil sudafricana, que en particular se unía en torno al Congreso Nacional Nativo Sudafricano, el cual presentaba narrativas en contra del imaginario colonial imperial, subrayando la necesidad de (y la posibilidad de) igualdad, a pesar de la diferencia (Plaatje, 1995). Ciertamente uno de los activistas más conocidos fue Sol Plaatje, cuyas críticas - por ejemplo, al hecho de que "el nativo sudafricano realmente no se consideraba a sí mismo como esclavo, sino como paria en su tierra natal" (Plaatje, 1995, p. 21)-desafiaron las bases del poder blanco y británico en el país (Boehmer, 2002). Incluso si Gladstone hubiera estado inclinado a descartar dicha crítica, 
también había europeos que se rehusaban a ver a los africanos como inferiores. Por ejemplo, durante el Congreso de los Nativos de Sudáfrica celebrado en Kimberley, en 1914, un orador fue Leyton Richards, ministro de la Congregación Británica que más tarde se convirtió en figura clave en el movimiento pacifista de Gran Bretaña (“The Rev. Leyton Richards”, 1948) y que hizo un llamado al establecimiento de un "orden social más noble" en Sudáfrica, un orden en el que "cada raza debe contribuir en su valor más pleno al conjunto social y en el que no debe haber ninguna barrera de color, raza o credo a través de los caminos de aquellos que escalarán hacia mejores cosas”. Se citó a Richards diciendo que "se alegraba de tener la oportunidad de hablar frente al Congreso, no como miembro de una raza superior (porque él no admitía que la diferencia implicara superioridad), sino como un hombre hablándoles a otros hombres” (“S. A. Native Congress”, 1914; el énfasis es mío). Dicha visión sobre la raza — de igualdad a pesar de la diferencia; de que la diferencia no implicaba jerarquía- se convirtió en parte de la base del movimiento internacional de los derechos humanos que modeló al siglo xx de manera profunda (Hoffmann, 2011). En Sudáfrica, por supuesto, se mezcló con el panafricanismo radical y el comunismo para desafiar las bases en las que se asentaba el gobierno del apartheid (Beinart, 2001). Sin embargo, incluso en la Sudáfrica de Herbert Gladstone, e incluso en 1914, hubo desafíos al tipo de visión condescendiente que él tenía sobre los africanos. Al parecer, Gladstone se negaba a escucharlos, optaba por ignorarlos o, simplemente, los desdeñaba. Tal vez si se hubiera conectado con la emergente sociedad civil en Sudáfrica -y hubiera logrado desarrollar algo más allá de la típica actitud colonial hacia los africanos- su optimismo respecto al país hubiera estado más justificado. En lugar de eso, Gladstone abandonó Sudáfrica en 1914 sin aceptar nunca el significado último de la aventura colonial-imperial que realizó, y en la que, a pesar de que desempeñó un papel relativamente menor, no dejó de ser un papel privilegiado.

Dirección institucional del autor:

Maynooth University

Maynooth, Co. Kildare, Ireland 


\section{Bibliografía}

AlLEN, J. (2003). Lost geographies of power. Malden: Blackwell.

AsHForTH, A. (1997). Lineaments of the political geography of state formation in twentieth-century South Africa. Journal of Historical Sociology, 10(2), 101-126. https://doi.org/10.1111/14676443.00033

Auld, J. W. (1975). The liberal pro-boers. Journal of British Studies, 14(2), 78-101. https://doi.org/10.1086/385672

BeInART, W. (2001). Twentieth-Century South Africa. Oxford, Nueva York: Oxford University Press.

BeInART, W. y Delius, P. (2014). The historical context and legacy of the natives Land Act of 1913. Journal of Southern African Studies, 40(4), 667-688. https://doi.org/10.1080/03057070.2014.930623

BOEHMER, E. (2002). Empire, the national, and the postcolonial, 18901920: Resistance in interaction. Oxford, Nueva York: Oxford University Press.

Bottomley, E.-J. (2016). Transnational governmentality and the "poor white" in early twentieth century South Africa. Journal of Historical Geography, (54), 76-86. https://doi.org/10.1016/j. jhg.2016.09.002

ButLER, J. (2010). Performative agency. Journal of Cultural Economy, 3(2), 147-161. https://doi.org/10.1080/17530350.2010.494117

Callinicos, L. (1985). Gold and workers, 1886-1924. Johannesburgo: Ravan Press.

Cox, K. R. (2002). Political geography: Territory, state, and society. Oxford: Blackwell.

Cox, K. R. (2016). Geographies, critical and Marxist, and lessons from South Africa. Human Geography, 9(3), 10-26.

Dubow, S. (1997). Colonial nationalism, the Milner kindergarten and the rise of "South Africanism", 1902-10. History Workshop Journal, (43), 53-85.

Evans, I. (2005). Racial violence and the origins of segregation in South Africa. En C. Elkins y S. Pedersen (Eds.), Settler colonialism in the twentieth century (pp. 183-202). Nueva York: Routledge.

Graham, L. V. (2012). State of Peril: Race and rape in South African literature. Nueva York: Oxford University Press.

HARCOURT, L. (2006). Loulou: Selected extracts from the journals of Lewis Harcourt (1880-1895) (Ed. P. Jackson). Madison: Farleigh Dickinson University Press.

Harris, J. (2010). The liberal empire and British social policy: Citi- 
zens, colonials, and indigenous peoples, circa 1880-1914. Histoire@Politique, 11(2),1-14.https://doi.org/10.3917/hp.011.0003 Harvey, D. (1982). The limits to capital. Oxford: Basil Blackwell.

Higginson, J. (2001). Hell in small places: Agrarian elites and collective violence in the Western Transvaal, 1900-1907. Journal of Social History, 35(1), 95-124. https://doi.org/10.1353/jsh.2001.0084 HofFmanN, S.-L. (2011). Human rights in the twentieth century. Cambridge, Nueva York: Cambridge University Press.

Hyslop, J. (2014). The strange death of liberal England and the strange birth of illiberal South Africa: British trade unionists, Indian labourers and Afrikaner rebels, 1910-1914. Labour History Review, 79(1), 97-120. https://doi.org/10.3828/lhr.2014.6

Jenkins, R. (1978). Asquith. Londres: Collins.

JENKINS, R. (1995). Gladstone. Londres: Macmillan.

Le May, G. H. (1965). British supremacy in South Africa, 1899-1907. Oxford: Clarendon Press.

Mallet, C. E. (1932). Herbert Gladstone: A Memoir. Londres: Hutchinson \& Co.

Mamdani, M. (1996). Citizen and subject: Contemporary Africa and the legacy of late colonialism. Londres: James Currey.

Marks, S. y Trapido, S. (1979). Lord Milner and the South African State. History Workshop, 8(1), 50-80. https://doi.org/10.1093/ $\mathrm{hwj} / 8.1 .50$

Massey, D. (1994). Space, place and gender. Cambridge: Polity Press. Massey, D. (2005). For space. Londres: SAGE.

Massey, D. y Allen, J. (Eds.). (1995). Geographical worlds (The shape of the world: Explorations in human geography). Oxford: Oxford University Press.

McClendon, T. V. (2010). White chief, Black lords: Shepstone and the colonial state in Natal, South Africa, 1845-1878. Rochester: University of Rochester Press.

O'Meara, D. (1996). Forty lost years: The apartheid state and the politics of the National Party, 1948-1994. Randburg: Ravan Press; Athens: Ohio University Press.

Painter, J. (2006). Prosaic geographies of stateness. Political Geography, 25(7), 752-774. https://doi.org/10.1016/j.polgeo.2006.07. 004

Plantje, S. T. (1995). Native life in South Africa before and since the European War and the Boer Rebellion. Randburg: Ravan Press.

PlatzKy, L. y Walker, C. (1985). The surplus people: Forced removals in South Africa. Johannesburgo: Ravan Press.

Posel, D. (1991). The making of apartheid, 1948-1961: Conflict and 
compromise. Oxford: Clarendon Press; Nueva York: Oxford University Press.

Raco, M. (2003). Governmentality, subject-building, and the discourses and practices of devolution in the UK. Transactions of the Institute of British Geographers, 28(1), 75-95. https://doi. org/10.1111/1475-5661.00078

S. A. Native Congress (3 de marzo de 1914). Diamond Fields Advertiser.

ScoTT, J. C. (1998). Seeing like a state: How certain schemes to improve the human condition have failed. New Haven: Yale University Press.

The Rev. Leyton Richards. (24 de agosto de 1948). The Times.

TRIPP, D. (1994). “Teachers" lives, critical incidents, and professional practice. International Journal of Qualitative Studies in Education, 7(1), 65-76. https://doi.org/10.1080/0951839940070105

TuFfNELL, S. (2015). Engineering inter-imperialism: American miners and the transformation of global mining, 1871-1910. Journal of Global History, 10(1), 53-76. https://doi.org/10.1017/S1740 022814000369

\section{Materiales de archivo:}

The National Archives (Gran Bretaña), CO551/53-55 (Gladstone a Harcourt, 14 y 15 de enero de 1914), CO551/54 (Gladstone a Loulou Harcourt, 22 de enero de 1914) y CO551/55 (Gladstone a Harcourt, 31 de enero de 1914).

British Library, documentos del visconde Gladstone, Manuscritos adicionales 45997 (Gladstone a Harcourt, 14 de julio de 1911), 46113 (Discurso de Gladstone en Ciudad del Cabo, 10 de julio de 1914) y 46118 (borrador mecanografiado de la autobiografía inédita de Gladstone). 
